\title{
An IL-13 inhibitor blocks the development of hepatic fibrosis during a T-helper type 2-dominated inflammatory response
}

\author{
Mónica G. Chiaramonte, ${ }^{1}$ Debra D. Donaldson, ${ }^{2}$ \\ Allen W. Cheever, ${ }^{3}$ and Thomas A. Wynn ${ }^{1}$ \\ ${ }^{1}$ Schistosomiasis Immunology and Pathology Unit, Immunobiology Section, Laboratory of Parasitic Diseases, \\ National Institute of Allergy and Infectious Diseases, National Institutes of Health, Bethesda, Maryland 20892, USA \\ ${ }^{2}$ Genetics Institute, Cambridge, Massachusetts 01810, USA \\ ${ }^{3}$ Biomedical Research Institute, Rockville, Maryland 20852, USA \\ Address correspondence to: Thomas A. Wynn, National Institutes of Health, Building 4/126, Bethesda, Maryland 20892-0425, USA. \\ Phone: (301) 496-4758; Fax: (301) 402-0077; E-mail: twynn@niaid.nih.gov. \\ Received for publication May 13, 1999, and accepted in revised form August 2, 1999.
}

In schistosomiasis, chronic parasite egg-induced granuloma formation can lead to tissue destruction and fibrosis, which causes much of the morbidity and mortality associated with this disease. Here we show the importance of IL-13 in the pathogenesis of schistosomiasis, and demonstrate, perhaps for the first time, the therapeutic efficacy of an IL-13 inhibitor, sIL-13R $\alpha 2-\mathrm{Fc}$, in the control of hepatic fibrosis. T-helper type 2 (Th2) cytokines dominate the immune response in mice infected with Schistosoma mansoni, yet the specific contributions of IL-13 and IL-4 to the development of fibrosis were not previously investigated. Our studies demonstrate that both cytokines play redundant roles in granuloma formation, which explains the ability of IL-4-deficient mice to form granulomas around eggs. More importantly, however, these studies demonstrate that IL-13 is the dominant Th2-type cytokine regulating fibrosis. IL-13 stimulated collagen production in fibroblasts, and procollagen I and procollagen III mRNA expression was decreased in sIL-13R $\alpha 2$-Fc-treated mice. Moreover, the reduction in fibrosis observed in IL-4-deficient mice was much less pronounced than that in SIL-13R $\alpha 2$-Fc-treated animals. Fibrosis is a major pathological manifestation of a number of allergic, autoimmune, and infectious diseases. Thus, our findings provide evidence that IL-13 inhibitors may be of general therapeutic benefit in preventing damaging tissue fibrosis resulting from Th2-dominated inflammatory responses.

J. Clin. Invest. 104:777-785 (1999).

\section{Introduction}

The development of fibrous tissue is part of the normal process of healing after injury. Nevertheless, in some circumstances, there is a destructive accumulation of excess collagen that interferes with the normal function of the affected tissue. Indeed, collagen synthesis and tissue scaring are the major pathological manifestations of a number of chronic and debilitating illnesses, including several autoimmune, allergic, and infectious diseases (1). Although there is a great deal of mechanistic information regarding the process of scar tissue formation $(2,3)$, there are still large gaps in our understanding of the role of inflammatory cells and cytokines in initiating the fibrotic process. In murine schistosomiasis, the pathology resulting from infection with the helminth parasite Schistosoma mansoni is predominantly caused by the host reaction to parasite eggs that are laid in the portal venous system and subsequently trapped in the liver and intestine (4). The associated egg-induced fibrosis can lead to portal hypertension, which causes much of the morbidity and mortality associated with this disease. Schistosomiasis is caused by several species of trematode worms and is believed to affect over 200 million people worldwide, causing between 500,000 and 800,000 deaths per year (5). Elucidating the mechanisms leading to tissue pathology and fibrosis may lead to more effective strategies for immunological intervention in this and a variety of chronic diseases.

In the murine model of schistosomiasis, several Th2associated cytokines, including IL-4, IL-5, IL-10, and IL13 , are induced after infection with S. mansoni (6-8) and contribute to many aspects of the host's immune response against the parasite (9-13). Indeed, knockout and cytokine ablation studies have clearly demonstrated an important role for Th2-type cytokines in granuloma formation, tissue eosinophilia, IgG1/IgE antibody production, and the development of hepatic fibrosis $(10,13$, 14). Because IL-4 is the primary cytokine driving the differentiation of $\mathrm{CD}^{+} \mathrm{T}$ cells into the Th2 subset $(15,16)$, it was predicted that much of the pathology associated with schistosome infection would be ameliorated by the elimination of IL-4. Nevertheless, IL-4 ablation experiments and studies with IL-4-deficient mice failed to demonstrate an indispensable role for this cytokine (8, $9,17,18)$. Indeed, these and related studies examining Th2 response development in IL-4-deficient mice demonstrated clearly that a significant, albeit diminished, Th2-type response can develop in the absence of IL-4 $(8,19,20)$. These findings suggest that IL-4 is not the sole mediator of egg-induced pathology, and that other cytokines are compensating and perhaps playing a more critical role in the pathogenesis of schistosomiasis. 
Because IL-13 shares many functional activities with IL-4 (21) and uses similar receptor subunits for signaling (22), it is possible that IL-13 plays an important role in schistosomiasis pathogenesis. With the recent development of IL-13 transgenic and knockout mice $(23,24)$, as well as soluble IL-13 antagonists (25), the unique functional activities of IL-13 are being delineated. Recent in vivo studies with several infectious disease $(20,23,24$, 26-29) and asthma models $(30,31)$ suggest that IL-13 possesses many important functional activities that are distinct from IL-4. The IL-13 receptor complex is composed of at least 3 distinct components, including the IL4 receptor, the low-affinity binding chain IL-13R $\alpha 1$, and the high-affinity binding chain IL-13R $\alpha 2(25,32-34)$. Recently, a soluble IL-13R $\alpha 2-F c$ fusion protein was prepared, and has been used successfully to neutralize IL-13 both in vitro (25) and in vivo (28-31). Because the fusion protein binds IL-13 with high affinity, but fails to neutralize IL-4, the protein provided an excellent tool to dissect the specific roles of IL-13.

In the present study, we used the IL-13 antagonist in wild-type (WT) and IL-4-deficient mice in order to dissect the contributions of IL-13 and IL-4 to the pathogenesis of schistosomiasis. In these studies, liver granuloma formation was examined in detail, focusing on eosinophil and mast cell recruitment, and the development of egg-induced fibrosis was quantified using biochemical, histological, and molecular techniques. Whereas the results from this study show that IL-13 and IL-4 exhibit some redundant activities in schistosomiasis pathogenesis, distinct functions for both cytokines were also clearly elucidated. The most important and novel finding was the observation that IL-13, not IL-4, was the major Th2-type cytokine driving type I and type III collagen mRNA production and hepatic fibrosis in infected mice. Thus, our findings provide evidence that an IL-13 inhibitor, such as sIL-13R $\alpha 2-F c$, may be of therapeutic benefit in preventing fibrosis associated with chronic infectious disease.

\section{Methods}

Animals, parasites, and antigen preparations. Six- to 8-week-old female C57BL/ 6 mice and IL-4-deficient mice (C57BL/6 background, 10th backcross) were obtained from Taconic Farms Inc. (Germantown, New York, USA) and were infected by percutaneous challenge. Cercariae of a Puerto Rican strain of S. mansoni (Naval Medical Research Institute, Bethesda, Maryland, USA) were obtained from infected Biomphalaria glabrata snails (Biomedical Research Instruments, Rockville, Maryland, USA). Soluble egg antigen (SEA) was purified from homogenized eggs, as previously described (9). The soluble IL-13 receptor $\alpha 2-\mathrm{Fc}$ fusion protein (sIL-13R $\alpha 2-\mathrm{Fc}$ ) and human IgG (control$\mathrm{Fc}$ ) were prepared as described previously (25) (provided by Genetics Institute Inc., Cambridge, Massachusetts, USA). Animals were treated by intraperitoneal injection in $0.5 \mathrm{~mL}$ PBS $(200 \mu \mathrm{g} /$ mouse per day) every other day, after the onset of egg production. As described previously, the control-Fc had no detectable effect on pathology or cytokine expression in infected mice (29). No mortality was observed among any groups of mice, and there were no signs of cachexia up to 8 weeks after infection.
Histopathology and fibrosis measurement. The sizes of hepatic granulomas were determined in histological sections stained by Wright's Giemsa stain (Histopath of America, Clinton, Maryland, USA). The percentages of eosinophils, mast cells, and other cell types were evaluated in the same sections. Parenchymal necrosis was scored on a scale of $0-4$, with 0 being the least extensive necrosis and 4 being the most extensive necrosis. The frequency of mast cells was also assigned on a similar scale, using a range of $0-4$. The number of schistosome eggs in the liver and gut and the collagen content of the liver, determined as hydroxyproline, were measured as described previously (9). Specifically, hepatic collagen was measured as hydroxyproline by the technique of Bergman and Loxley after hydrolysis of a 200 -mg portion of liver in $5 \mathrm{~mL}$ of $6 \mathrm{~N} \mathrm{HCl}$ at $110^{\circ} \mathrm{C}$ for 18 hours (35). The increase in hepatic hydroxyproline was positively related to egg numbers in all experiments (data not shown), and hepatic collagen is reported as the increase above normal liver collagen in micromoles per 10,000 eggs: (infected liver collagen - normal liver collagen)/liver eggs $\times$ $10^{-4}$. Fibrosis was also scored histologically using sections stained with picrosirius red. All granulomas within each section were scored for picrosirius red density on a scale of $1-4$, and a second measurement of area involved was also determined using the same scale. The total fibrosis score was determined by multiplying the density and area for each granuloma (a score of 16 would be the maximum). An average of 30 granulomas per mouse were included in all analyses. To control for consistency, the same individual scored all histological features and had no knowledge of the experimental design.

Isolation and purification of RNA and RT-PCR. Liver tissues were homogenized in RNA-STAT 60 (Tel-Test Inc., Friendswood, Texas, USA) using a tissue polytron (Omni International Inc., Warrenton, Virginia, USA), and total RNA was extracted following the recommendations of the manufacturer. An RT-PCR procedure was used to determine relative quantities of mRNA for several cytokine genes after reverse transcription of $1 \mu \mathrm{g}$ of RNA as described (36). The primers and probes for all genes were published previously $(13,36,37)$. The PCR cycles used for each cytokine were as follows: IL-4 (33), IL-5 (31), IFN- $\gamma(29)$, TNF- $\alpha$ (34), procollagen I (26), procollagen III (22), TGF- $\beta_{1}(34)$, TGF- $\beta_{2}$ (34), and hypoxanthine-guanine phosphoribosyl transferase (HPRT) (23). The amount of PCR product was determined by comparing the ratio of cytokine-specific signal density with that of HPRT-specific signal density for individual samples. Arbitrary densitometric units for individual samples were subsequently multiplied by a factor of 100 .

In vitro cultures. Mesenteric lymph node (MLN) cells and spleens were extracted from the mice, and single-cell suspensions were prepared as previously described (9). Cells were placed in RPMI-1640 medium $\left(3 \times 10^{6} / \mathrm{mL}, 1\right.$ $\mathrm{mL}$ ) supplemented with $10 \%$ FCS, $2 \mathrm{mM}$ glutamine, 100 $\mathrm{U} / \mathrm{mL}$ penicillin, $100 \mu \mathrm{g} / \mathrm{mL}$ streptomycin, $25 \mathrm{mM}$ HEPES, $1 \mathrm{mM}$ sodium pyruvate, $0.1 \mathrm{mM}$ nonessential amino acids, and $50 \mu \mathrm{M} 2-\mathrm{ME}$ at $37^{\circ} \mathrm{C}$ in $5 \% \mathrm{CO}_{2}$. Cells were stimulated with SEA $(20 \mu \mathrm{g} / \mathrm{mL})$, and supernatants were collected after 72 hours. Additional SEA-stimulated cultures were also treated with $50 \mu \mathrm{g} / \mathrm{mL}$ of anti-CD4 $\mathrm{mAb}(\mathrm{GK} 1.5)$. IL-4, IL-5, IL-10, and IFN- $\gamma$ were measured 
using specific sandwich ELISA (9). IL-13 levels were measured using murine IL-13 ELISA kits (R\&D Systems Inc., Minneapolis, Minnesota, USA).

Western blot detection of collagen I. 3T3 fibroblasts were cultured in RPMI-1640 medium supplemented as described above. Confluent cells were plated in 24-well plates (500,000 cells $/ \mathrm{mL})$ and stimulated with IL-4 $(1,000 \mathrm{U} / \mathrm{mL})$ or rIL-13 (20 ng/mL; R\&D Systems Inc.). Culture supernatants were collected to analyze secreted collagen I. Cells were washed once with PBS and lysed with SDS-PAGE sample buffer. Cell lysates and culture supernatants were submitted to electrophoretic separation in 6\% Tris-glycine gels (Novel Experimental Technology, San Diego, California, USA), using reducing conditions, and were transferred to nitrocellulose membranes (Schleicher \& Schuell Inc., Keene, New Hampshire, USA). Blots were probed with rabbit IgG anti-mouse type I collagen (BIODESIGN International, Kennebunk, Maine, USA), and peroxidase-labeled anti-rabbit IgG (Amersham Pharmacia Biotech, Piscataway, New Jersey, USA) was used as a second antibody. The bands were viewed using a Western blot chemiluminescence reagent (NEN Life Science Products Inc., Boston, Massachusetts, USA). To confirm the identity of the collagen bands, cell lysates were treated with $0.5 \mathrm{mg} / \mathrm{mL}$ of collagenase (Boehringer Mannheim Biochemicals, Indianapolis, Indiana, USA) in PBS supplemented with $1 \mathrm{mM}$ $\mathrm{CaCl}_{2}$ and $1 \% \mathrm{FCS}$, for 1 hour at $37^{\circ} \mathrm{C}$. A purified rat collagen I consisting of the $\beta 11(\mathrm{I}), \beta 12(\mathrm{I})$ and $\alpha 1(\mathrm{I}), \alpha 2(\mathrm{I})$ collagens was obtained from Sigma Chemical Co. (St. Louis, Missouri, USA) and used as a control.

Statistics. Granuloma size and hepatic fibrosis (adjusted for egg number) decrease with increasing intensity of infection (worm pairs) in these and previous experiments. These variables were, therefore, compared by analysis of covariance, using the log of total liver eggs as the covariate and the log of hydroxyproline per egg. Variables that did not change with infection intensity were compared by 1-way ANOVA or by Student's $t$ test. Worm and egg burdens were evaluated using the Wilcoxon rank sum test, with Fisher's combination test applied when results from 2 or more experiments were pooled.
Changes in cytokine mRNA and values for secreted cytokine proteins were determined by ANOVA. In all cases, results were considered significant for $P<0.05$.

\section{Results}

Treatment with sIL-13RO2-Fc significantly reduces hepatic fibrosis in S. mansoni-infected mice. To compare the regulatory roles of IL-4 and IL-13 in the pathogenesis of schistosomiasis, we infected C57BL/6 WT and IL-4-deficient mice percutaneously with $25 \mathrm{~S}$. mansoni cercariae. Separate groups of animals were treated with either sIL-13R $\alpha 2-F c$ or with control-Fc, as described in Methods. The treatments began on week 5 , at the start of egg laying. All animals were sacrificed 8 weeks after infection and examined for several parasitologic and immunologic parameters. All 4 groups of mice harbored similar worm burdens, and tissue eggs produced per worm pair did not vary among the groups (Table 1). At 8 weeks after infection, the time of the peak tissue response (38), WT mice showed no significant change in granuloma size as a result of IL-13 blockade (Figure 1a). Interestingly, control-Fc-treated IL-4-deficient mice also failed to show a reduced granulomatous response, and, in fact, granulomas were significantly larger in these mice. In striking contrast to these observations, the IL-4-deficient mice displayed a markedly reduced granulomatous response when IL-13 was inhibited (Figure 1a, far right). Indeed, the double IL-4-deficient/sIL-13R $\alpha 2-F c$-treated mice displayed, on average, a $40-50 \%$ reduction in granuloma volume when compared with either control or sIL13R $\alpha 2-F c$-treated WT animals, and more than a $75 \%$ reduction when compared with control-Fc-treated IL4-deficient mice.

The cellular composition of the lesions was also evaluated in Giemsa-stained liver sections. IL-4-deficient mice displayed a marked reduction in granuloma-associated mast cells (Table 1). In contrast, there was no change in mast cell numbers by IL-13 inhibition alone, and IL-13 blockade had no additional effect on the already highly reduced numbers of mast cells in IL-4-deficient mice. Somewhat similar, yet distinct, findings were observed when granuloma-associated eosinophils were evaluated

Table 1

Parasitological measurements in infected mice

\begin{tabular}{|c|c|c|c|c|c|c|c|}
\hline \multirow[b]{2}{*}{ Group } & & \multicolumn{3}{|c|}{ Parasite recovery } & \multicolumn{3}{|c|}{ Histological features of granulomas } \\
\hline & & $\begin{array}{c}\text { Worm } \\
\text { pairs }\end{array}$ & $\begin{array}{c}\text { Total } \\
\text { worms }\end{array}$ & $\begin{array}{l}\text { Eggs/ } \\
\text { worm pair } \\
(\times 1000)\end{array}$ & $\begin{array}{c}\text { Mast cells } \\
(0-4)^{A}\end{array}$ & $\begin{array}{c}\text { Parenchymal } \\
\text { necrosis } \\
(0-4)^{\mathrm{B}}\end{array}$ & $\begin{array}{c}\text { Fibrosis } \\
\text { sirius density }\end{array}$ \\
\hline A & $\begin{array}{l}\text { WT } \\
\text { Control-Fc } \\
\text { (N) }\end{array}$ & $\begin{array}{c}5.25 \\
\pm 0.57 \\
(20)\end{array}$ & $\begin{array}{c}12.7 \\
\pm 1.09 \\
(20)\end{array}$ & $\begin{array}{c}5.80 \\
\pm 0.52 \\
(20)\end{array}$ & $\begin{array}{c}1.60 \\
\pm 0.35 \\
(10)\end{array}$ & $\begin{array}{c}1.70 \\
\pm 0.35 \\
(10)\end{array}$ & $\begin{array}{c}10.50 \\
\pm 1.29 \\
(10)\end{array}$ \\
\hline B & $\begin{array}{l}\text { WT } \\
\text { IL-13R } \alpha 2-F c \\
(N)\end{array}$ & $\begin{array}{c}5.58 \\
\pm 0.76 \\
(19)\end{array}$ & $\begin{array}{c}13.9 \\
\pm 1.59 \\
(19)\end{array}$ & $\begin{array}{c}6.00 \\
\pm 0.39 \\
(19)\end{array}$ & $\begin{array}{c}2.22 \\
\pm 0.38 \\
(9)\end{array}$ & $\begin{array}{c}0.56^{\mathrm{E}} \\
\pm 0.23 \\
(9)\end{array}$ & $\begin{array}{c}3.66^{\mathrm{E}} \\
\pm 0.80 \\
(9)\end{array}$ \\
\hline C & $\begin{array}{l}\text { IL-4 KO } \\
\text { Control-Fc } \\
\text { (N) }\end{array}$ & $\begin{array}{c}4.50 \\
\pm 0.56 \\
(14)\end{array}$ & $\begin{array}{c}12.0 \\
\pm 1.13 \\
(14)\end{array}$ & $\begin{array}{c}6.96 \\
\pm 0.43 \\
(14)\end{array}$ & $\begin{array}{c}0.10^{\mathrm{D}} \\
\pm 0.09 \\
(10)\end{array}$ & $\begin{array}{c}1.56 \\
\pm 0.35 \\
(10)\end{array}$ & $\begin{array}{c}11.50 \\
\pm 1.47 \\
(10)\end{array}$ \\
\hline $\mathrm{D}$ & $\begin{array}{l}\text { IL-4 KO } \\
\text { IL-13R } \alpha 2-\mathrm{Fc} \\
(\mathrm{N})\end{array}$ & $\begin{array}{c}4.05 \\
\pm 0.45 \\
(19)\end{array}$ & $\begin{array}{c}11.4 \\
\pm 0.90 \\
(19)\end{array}$ & $\begin{array}{c}5.68 \\
\pm 0.54 \\
(18)\end{array}$ & $\begin{array}{l}0.0^{\mathrm{D}} \\
\pm 0 \\
(9)\end{array}$ & $\begin{array}{c}0.87^{\mathrm{E}} \\
\pm 0.48 \\
(9)\end{array}$ & $\begin{array}{c}2.44 \mathrm{E} \\
\pm 0.85 \\
(9)\end{array}$ \\
\hline
\end{tabular}

(N) Number of mice. All data are means for each group \pm 1 SEM. ${ }^{A}$ Mast cells within granulomas (scale $0-4$, none to numerous). ${ }^{\mathrm{B} P a r e n c h y m a l ~ n e c r o s i s ~ w a s ~ s c o r e d ~ f r o m ~}$ absent $(0)$ to most severe (4). CFibrosis as assessed by picrosirius staining of liver sections: Total area (1-4+) X Density (1-4+). ${ }^{D}$ Means significantly different from those in group $A$. ${ }^{\mathrm{N}}$ Means significantly different from those in group $\mathrm{A}$ and $\mathrm{C} ; P<0.05$. 
a

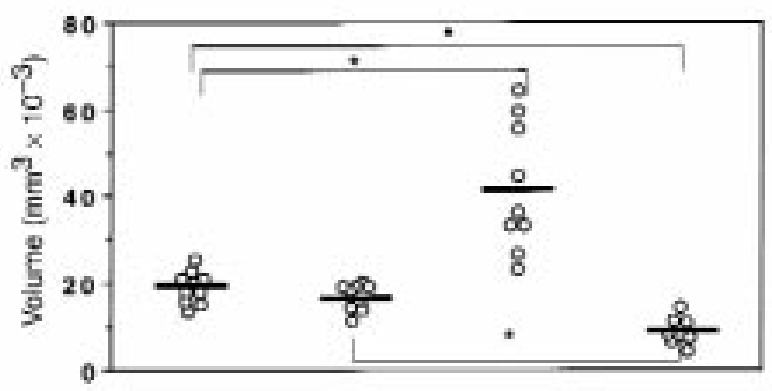

b

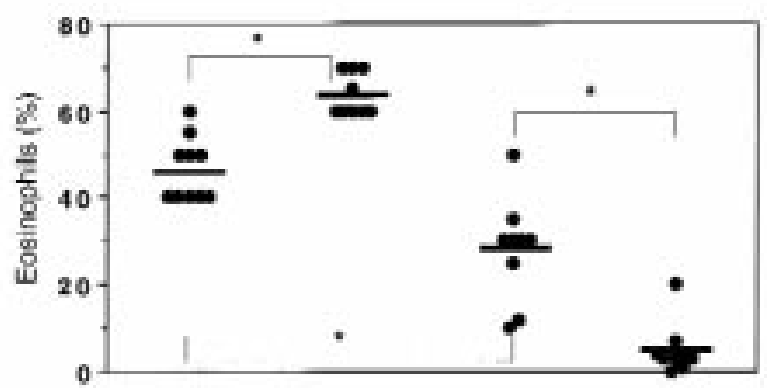

c

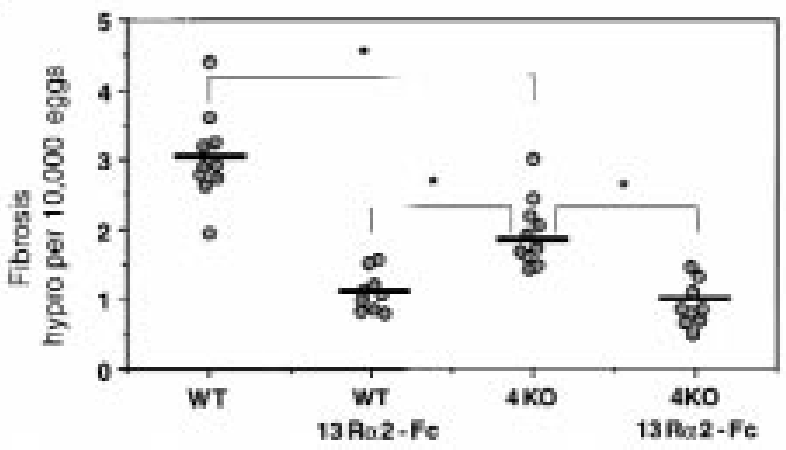

Figure 1

Characterization of the roles of IL-4 and IL-13 in schistosomiasis pathogenesis. C57BL/ 6 WT and IL-4-deficient (4KO) mice were sacrificed 8 weeks after infection to evaluate the size of liver granulomas (a), tissue eosinophilia (b), and hepatic fibrosis (c). Separate groups of mice were treated with control-Fc or sIL-13R $\alpha 2-\mathrm{Fc}$ as described in Methods. The data shown are measurements from individual mice. The bars designate the means for each group. Significant comparisons are indicated by asterisks. Similar data were reproduced in 3 separate studies.

(Figure 1b). Here, the numbers of eosinophils were increased from $46 \%$ to $64 \%$ in WT mice by IL-13 blockade, and were significantly decreased (28\%) as a consequence of IL-4 deficiency. Despite the apparent contrasting roles for IL-13 and IL-4 in the tissue eosinophilia, an even more striking combined inhibitory effect was observed when the IL-4-deficient mice were treated with the IL-13 inhibitor. In these mice, the average number of granuloma eosinophils was below $10 \%$. Finally, there was no change in the degree of parenchymal or egg-associated liver necrosis in the WT mice, compared with the IL4-deficient animals, whereas both sIL-13R $\alpha 2-F c-t r e a t e d$ WT and IL-4-deficient groups showed marked reductions in overall parenchymal necrosis (Table 1).

Perhaps most importantly, the sIL-13R $\alpha 2$-Fc treatment significantly reduced the collagen content of liver granulomas in WT mice, as assessed in tissue sections stained with picrosirius red (Table 1). In contrast, infected IL-4-deficient mice showed no detectable change in granuloma collagen deposition by microscopic analysis. Interestingly, there appeared to be no combined or synergistic role for IL-13 and IL-4 in this parameter, because there was no significant difference between sIL-13R $\alpha 2$ Fc-treated WT and IL-4-deficient mice. Finally, the extent of hepatic fibrosis was also measured by the assessment of hydroxyproline levels (Figure 1c), which is more quantitative than the histological techniques described above. The soluble IL-13 antagonist alone markedly decreased liver hydroxyproline levels, whereas the IL-4 deficiency resulted in a significantly less pronounced reduction. The dual IL-4/IL-13 deficiency failed to reduce hydroxyproline to levels below that already observed in the sIL-13R $\alpha 2$-Fc-treated WT mice. Together, these data demonstrate that IL-13 is the dominant Th2-associated cytokine responsible for the development of hepatic fibrosis in murine schistosomiasis.

Th2-type cytokine production is reduced in IL-4-deficient mice but unaffected by IL-13 inhibition. Whereas it is well known that IL-4 is the primary cytokine driving CD4 $4^{+} \mathrm{Th} 2$ cell development $(15,16)$, the role of IL-13 in the generation and maintenance of Th2-type responses remains controversial and may be influenced by both host genetics and the infectious disease model under study $(24,27,29)$. Therefore, to determine whether the sIL-13R $\alpha 2$ $\mathrm{Fc}$-induced changes in liver pathology were generated by alterations in the Th1/Th2 cytokine balance, we isolated MLNs and spleens from infected mice, prepared single-cell suspensions, and restimulated the cultures in vitro with parasite antigens. Additional cell cultures were exposed to parasite antigens in the presence of anti-CD4 $\mathrm{mAb}$ to determine whether cytokine production was dependent upon a $\mathrm{CD} 4^{+} \mathrm{T}$-cell response. Culture supernatants were analyzed by ELISA for IL-4, IL-13, IL-5, IL-10, and IFN- $\gamma$. As might be predicted (9), mesenteric (Figure 2) and splenic cultures (data not shown) prepared from WT mice displayed a highly polarized Th2-type cytokine response. They produced high levels of IL-4, IL-5, IL-10, and IL-13 in response to SEA stimulation, and little or no IFN- $\gamma$. IL4-deficient mice, in contrast, showed a more mixed Th1/Th2-type profile. Indeed, a significant SEA-specific IFN- $\gamma$ response was detected in IL-4-deficient mice, which is consistent with previous studies $(17,18)$. IL-13, IL-10, and, to a lesser extent, IL-5 were also detected in these animals, although the levels of these cytokines were markedly decreased when compared with WT mice. The maintenance of the low but significant IL-4-independent IL-13 response likely explains the marked granulomatous response that is maintained in the absence of IL-4 (Figure 1). Importantly, despite its marked inhibitory effect on hepatic fibrosis, sIL-13R $\alpha 2-\mathrm{Fc}$ had no significant effect on Th1 or Th2-type cytokine responses in WT and IL-4-deficient mice. It should also be noted that in all cases, cytokine production was highly dependent on a $\mathrm{CD} 4^{+} \mathrm{T}$ cell response, because little or no cytokine expression was detected in any of the anti-CD4 mAb-treated SEA-stimulated cultures.

Th1/Th2-type cytokine $m R N A$ expression in the granulomatous livers of IL-4-deficient and SIL-13R $\alpha 2-F c$-treated mice. To determine whether a similar pattern of cytokine expres- 

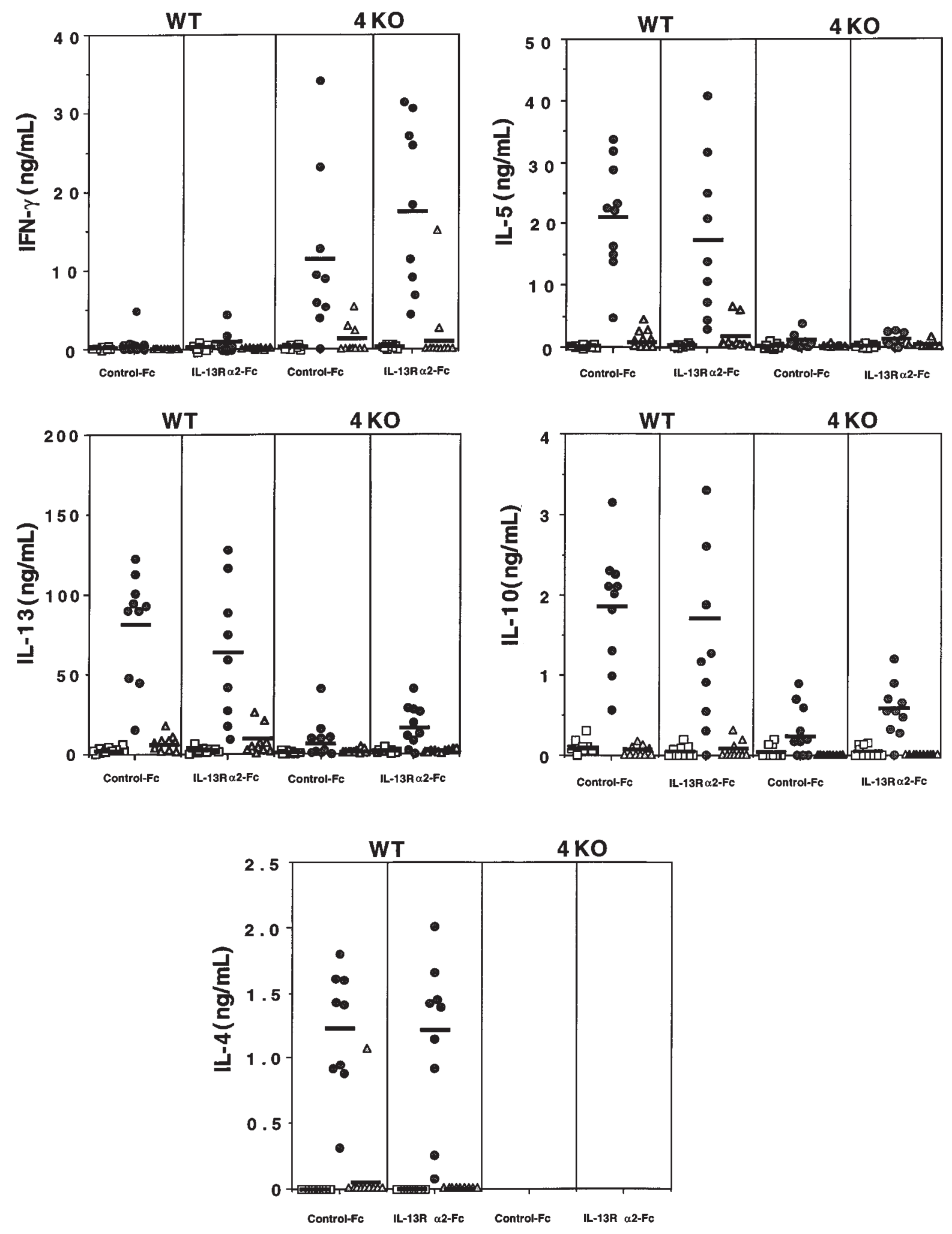

Figure 2

The Th1/Th2-type cytokine profile is unaffected by sIL-13R $\alpha 2-F c$ treatment. Infected C57BL/6 WT and IL-4-deficient (4KO) mice were treated with control-Fc or sIL-13R $\alpha 2-\mathrm{Fc}$ as described in Methods MLN cells were isolated from individual mice, and single-cell suspensions were prepared $\left(3 \times 10^{6}\right.$ cells per well in 24 -well plates) and stimulated with medium (squares), SEA at $20 \mu \mathrm{g} / \mathrm{mL}$ (circles), or SEA and $50 \mu \mathrm{g} / \mathrm{mL}$ of antiCD4 mAb (triangles). All cytokines were assayed in culture supernatants by ELISA 72 hours after stimulation, as described in Methods. The symbols represent values for individual mice, and the bars indicate the means within each group. 
sion was observed in vivo at the site of granuloma formation, we isolated liver mRNA from the various groups of mice at 8 weeks after infection and performed quantitative RT-PCR. Infected WT mice displayed a strong Th2-type cytokine mRNA profile, showing marked increases in IL-4, IL-13, IL-5, and IL-10 mRNA (Figure $3)$. The WT mice also showed modest increases in the expression of IFN- $\gamma$ mRNA, which was consistent with previous observations (13). In contrast to these findings, IL-13 and IL-5 mRNA levels were much lower in IL4 -deficient mice, whereas IL-10 and TNF- $\alpha$ mRNA increased, and IFN- $\gamma$ mRNA expression did not change significantly. Again, similar to the in vitro results obtained from MLN and splenocyte cultures, IL-13 blockade had no significant effect on the pattern of cytokine mRNA expression in WT and IL-4-deficient mice. There was, however, a modest increase in IL-10 mRNA levels in IL-4-deficient mice treated with the sIL$13 \mathrm{R} \alpha 2-\mathrm{Fc}$, although this is unlikely to explain the decreases in fibrosis, because highly divergent levels of IL-10 were detected in sIL-13R $\alpha 2$-Fc-treated WT mice, compared with IL-4-deficient mice, yet a similar decrease in fibrosis was observed. TGF- $\beta_{1}$ and TGF- $\beta_{2}$ mRNA expression was also examined in the granulomatous tissues; however, no significant differences were observed in infected IL-4-deficient mice or in animals treated with sIL-13R $\alpha 2-\mathrm{Fc}$ (data not shown).
Procollagen I and procollagen III $m R N A$ levels are reduced in the livers of $s I L-13 R \alpha 2-F c$-treated mice but are unaffected by $I L-4$ deficiency. The in vitro and in vivo cytokine studies described above suggested that the antifibrotic effect of sIL-13R $\alpha 2-F c$ was unlikely to be explained by changes in Th1- or Th2-type cytokine expression. Therefore, in subsequent experiments, we investigated the patterns of procollagen I (Col I) and procollagen III (Col III) mRNA expression to determine whether the sIL-13R $\alpha 2$ Fc-induced reduction in fibrosis was accompanied by direct changes in the expression of these 2 important collagen-producing genes (13). Here, IL-13 blockade significantly reduced Col I and Col III mRNA expression in both WT and IL-4-deficient mice (Figure 4). There was no change in the infection-induced levels of Col I and Col III mRNAs in IL-4-deficient mice compared with WT, and when compared with sIL-13R $\alpha 2$-Fc-treated WT mice, there was no further reduction in similarly treated IL-4-deficient mice.

IL-13 stimulates collagen production in mouse 3 T3 fibroblasts. Having shown that IL-13 blockade in vivo significantly reduced Col I and Col III mRNA expression in the liver of infected WT and IL-4-deficient mice, we wanted to determine whether IL-13 would directly stimulate collagen deposition in fibroblasts. To answer this question, we examined the induction of type I collagens in murine $3 \mathrm{~T} 3$ fibroblasts using Western blotting. As shown in Fig-
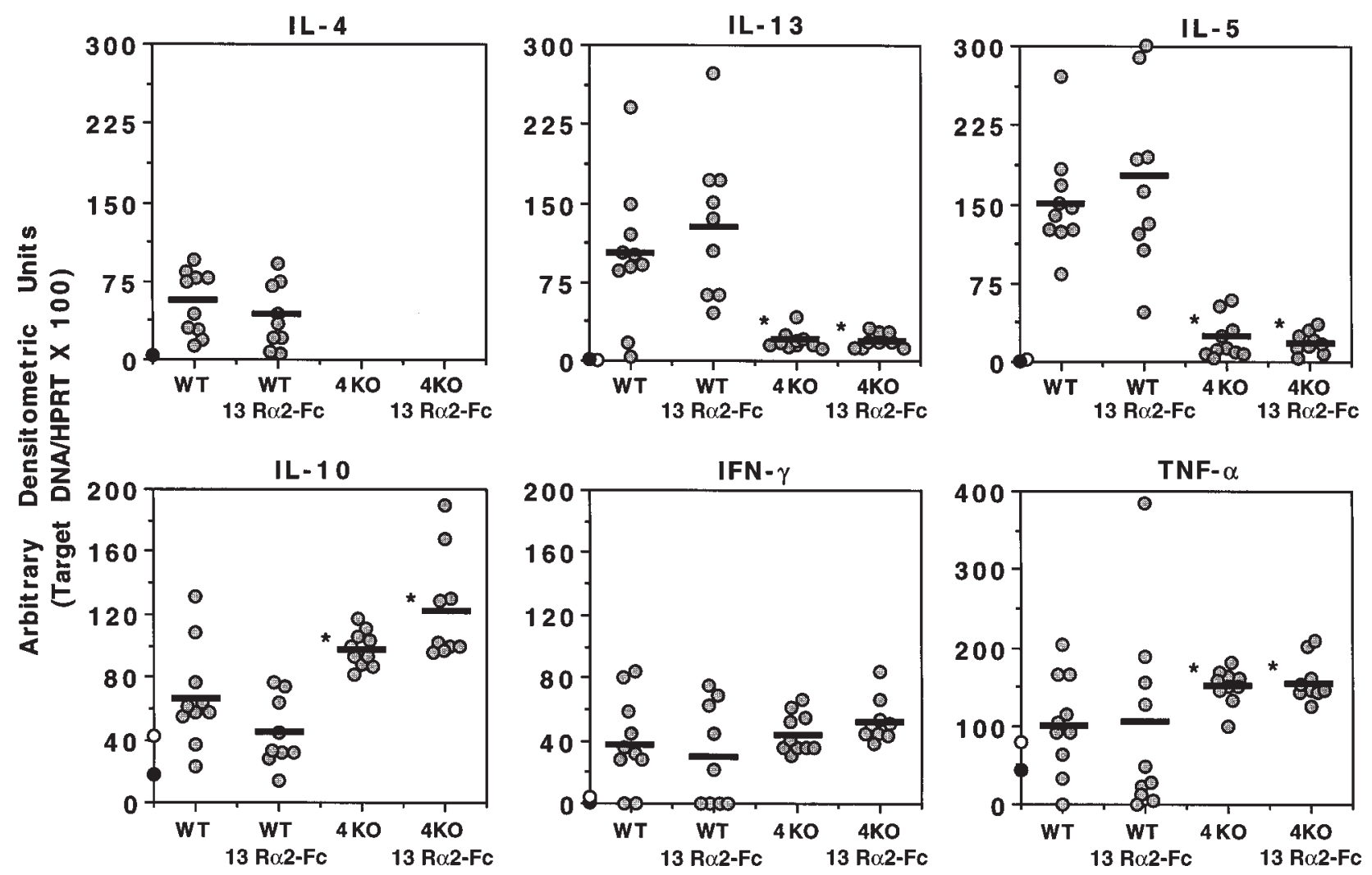

Figure 3

Th2-type cytokine mRNA expression is reduced in the livers of infected IL-4-deficient mice, but is unaffected by IL-13 blockade. Infected and treated C57BL/6 WT and IL-4-deficient (4KO) mice were sacrificed on week 8 after infection, and liver specimens were prepared for RT-PCR. The data shown are the individual values of 9-10 animals per group, and the bars indicate the average within each group. The average values from 5 uninfected WT (filled circles) and 5 uninfected IL-4-deficient mice (open circles) are shown on the $y$-axis for each cytokine. All data were reproduced in a second study. ${ }^{*}$ Data are significantly different from the WT control-Fc group, as determined by ANOVA $(P<0.05)$. 


\section{Figure 4}

Collagen I and collagen III mRNA expression is reduced in the livers of infected sIL-13R $\alpha 2-F c$-treated mice, but is unaffected by IL-4 deficiency (4KO). RT-PCR analysis was performed for collagen I and III as described in the legend to Figure 3. These data were reproduced in a separate study. a



b

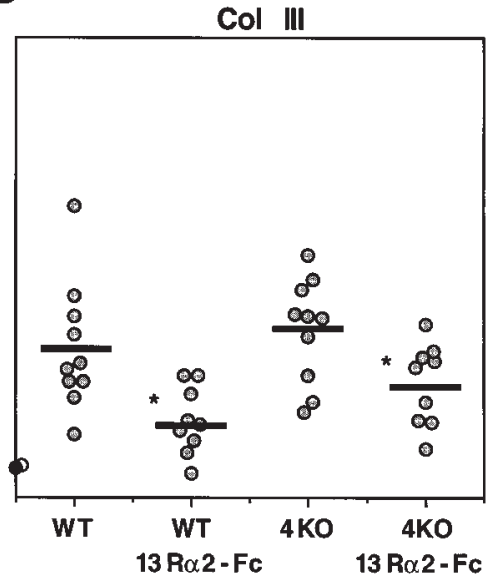

ure 5, IL-13 induced collagen synthesis 48 hours after exposure. Minimal type I collagen was detected in unstimulated cells (Figure 5a, lane 1) and at earlier time points in the cytokine-activated cultures (data not shown). IL-4 also induced collagen I synthesis (lane 2), and high levels of secreted collagen were easily detectable in the supernatants obtained from both cytokine-stimulated cultures (data not shown). We observed no significant difference in the ability of IL-4 and IL-13 to stimulate collagen I expression. The specificity of the reaction was confirmed by using purified collagen type I (lane 5), and bacterial collagenase treatments showed that the antibodies were specific for collagen type I, including the $\alpha$ and $\beta$ chains (data not shown).

\section{Discussion}

A $\mathrm{CD}^{+}$Th2-type cytokine pattern dominates the immune response in mice infected with $S$. mansoni $(6,7)$. Previous IL-4 depletion studies and experiments with IL4-deficient mice, however, failed to show an indispensable role for this cytokine in the pathogenesis of schistosomiasis $(8,9,17,18)$. Indeed, whereas a partial reduction in fibrosis was observed in some studies (9), egg-induced granuloma formation could proceed in the complete absence of IL-4 $(8,17,18)$. In contrast to these observations, granuloma formation and the development of hepatic fibrosis was severely impaired in Stat6-deficient mice (10), which display a major defect in the production of several Th2-associated cytokines (39). IL-4 and IL-13 both activate the Stat 6 signaling pathway. Therefore, the apparent differences in pathology observed between infected IL-4-deficient and Stat6-deficient mice may be explained by IL-13. Nevertheless, the distinct contribu-

\section{Figure 5}

IL-13 induces type I collagen synthesis in murine $3 \mathrm{~T} 3$ fibroblasts. Cells were stimulated with media (lane 1), rlL-4 at 1,000 U/mL (lane 2), or rlL-13 at $20 \mathrm{ng} / \mathrm{mL}$ (lanes 3 and 4) for 48 hours. Total cell lysates were separated on 6\% SDS-PAGE under reducing conditions, were transferred to nitrocellulose membrane, and were probed with rabbit IgG anti-mouse type I collagen. The anti-collagen type I antibody identifies purified collagen type I, including $\beta 11(I), \beta 12(I)$ and $\alpha 1(I), \alpha 2(I)$ collagen, which were separated in lane 5 (a). Assay specificity was confirmed by digestion with collagenase in separate experiments. (b) Densitometric values (arbitrary pixel units). tions of IL-4 and IL-13 in disease progression cannot be discerned from studies in Stat6, IL-4R $\alpha$, or IL-4-deficient mice alone. In this study, we used a potent inhibitor of IL13 in infected WT and IL-4-deficient mice, and demonstrate that IL-13 and IL-4 exhibit redundant, as well as unique, roles in the pathogenesis of schistosomiasis.

Several studies have shown that Th2-type cytokine responses can develop in vivo in the absence of IL-4 or the IL-4 receptor $(19,28)$, which is consistent with our findings that the levels IL-13, IL-10, and IL-5 were much reduced, but still significantly detectable in MLNs (Figure 2 ) and livers (Figure 3) of infected IL-4-deficient mice. Their production was also highly dependent on a $\mathrm{CD}^{+}{ }^{+} \mathrm{T}-$ cell response (Figure 2), further indicating that a conventional Th2-type response was established. These findings provide evidence that whereas maximal IL-13 expression is dependent on IL-4, the continued production of IL-13 might explain the maintenance of a significant granulomatous response in the absence of IL-4 $(8,17,18)$. Indeed, whereas blocking IL-13 alone had no effect on granuloma size in WT mice, inhibiting the residual IL-13 in IL-4-defi-

a

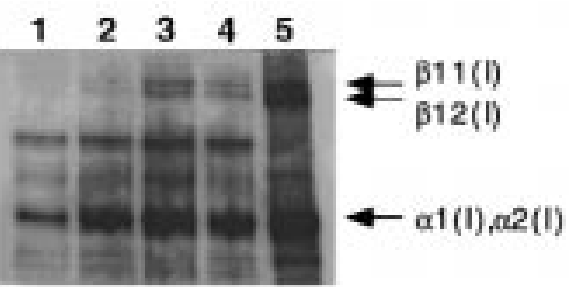

b

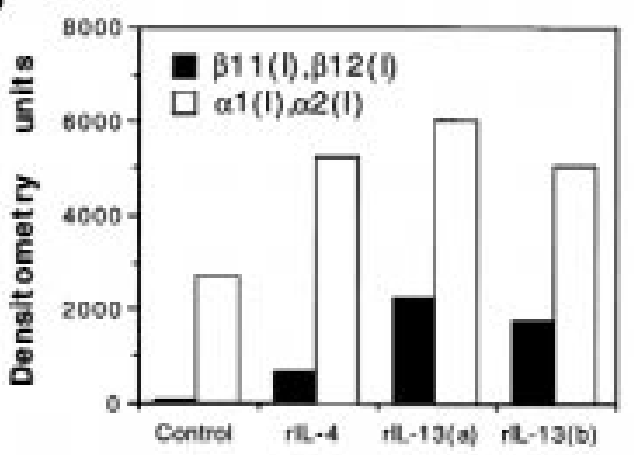


cient mice resulted in a marked and highly significant reduction in granuloma volume (Figure 1a). These findings demonstrate that IL-4 and IL-13 are both sufficient to mediate granuloma development, and formally explain the maintenance of granulomatous inflammation in IL4-deficient mice $(10,18)$. Similar findings were also reported in the pulmonary granuloma model, where the simultaneous depletion of IL-4 and IL-13 reduced granuloma formation to a much greater extent than either treatment alone (29). Because granulomas serve an important host-protective role by walling off potentially lethal hepatotoxins released by the eggs (40), the host may have evolved redundant mechanisms for granuloma formation in order to ensure a favorable host-parasite relationship.

Whereas these observations clearly demonstrate that IL4 and IL-13 actively participate in granuloma formation, unique roles for both cytokines in mast cell recruitment, tissue eosinophilia, and, most importantly, the generation of hepatic fibrosis were revealed. Histological examinations of liver sections from infected mice demonstrated that IL-13 is not required for mast cell (Table 1) or eosinophil (Figure 1b) differentiation and recruitment, because granulomas of sIL-13R $\alpha 2-F c$-treated WT mice showed no decrease in either cell type. In fact, eosinophil numbers were increased in the lesions of IL-13-inhibited WT mice (Figure 1b), suggesting that IL-13 may partially antagonize this effect in the presence of IL-4. In contrast, mast cells were almost completely absent from the lesions in IL-4-deficient mice, and eosinophils were decreased by over $50 \%$. Interestingly, IL- 13 appears to mediate the tissue eosinophilia in IL-4-deficient mice, because eosinophils were reduced to below $10 \%$ in the IL-4-deficient/sIL-13R $\alpha 2$-Fc-treated animals. Nevertheless, these data indicate that IL-4 is the dominant cytokine responsible for the development of eosinophil and mast cell populations within granulomas.

Probably the most important advance from this study was the finding that hepatic fibrosis could be blocked by sIL-13R $\alpha 2-F c$. Indeed, microscopic (Table 1), biochemical (Figure 1c), and molecular techniques (Figure 4) all indicated that IL-13, not IL-4, plays the major role in the development of egg-induced liver fibrosis. Previous studies showed that perturbations in the Th1/Th2 cytokine balance can significantly effect the extent of tissue fibrosis in S. mansoni-infected mice (13). Nevertheless, this study suggests that the effects of sIL-13R $\alpha 2-\mathrm{Fc}$ were not mediated through a skewing of the Th-cell cytokine response. Blocking IL-13 had no significant effect on the production of IFN- $\gamma$, IL-4, IL-5, IL-10, or IL-13 by MLN (Figure 2) or spleen cells in vitro, and there was also no change in cytokine mRNA expression in vivo at the site of lesion formation (Figure 3). In contrast to these observations, IL-4-deficient mice displayed an increased IFN$\gamma$ response in the draining lymph nodes (Figure 2) and decreased IL-5 and IL-13 expression in the lymph nodes (Figure 2) and liver (Figure 3). Thus, the less-pronounced reduction in fibrosis detected in IL-4-deficient mice by hydroxyproline analysis (Figure 1c) may be attributable to decreased IL-13 production. The fact that IL-4 production was unaffected by IL-13 blockade, yet fibrosis was maximally reduced in these animals, emphasizes the important role played by IL-13. Indeed, sIL-13R $\alpha 2$ Fc-treated IL-4-deficient mice showed little additional decrease in hydroxyproline levels (Figure 1c) and no difference in collagen I or collagen III mRNA expression (Figure 4) over that observed in similarly treated WT mice. There was also no change in collagen I or collagen III mRNA expression in control-Fc-treated IL-4-deficient mice when compared with WT animals, further deemphasizing the contribution of IL-4. It is also important to note that whereas the fibrosis scores by histology (Table 1) and collagen I and collagen III mRNA data (Figure 4) do not agree completely with the sensitive hydroxyproline results reported for the IL-4-deficient mice (Figure 1), they are completely consistent with the published and controversial role of this cytokine in schistosomiasis pathogenesis. Indeed, some studies reported a partial role for IL-4 in the generation of egginduced fibrosis (9), whereas a related study demonstrated no contribution at all (18). Therefore, the fact that the IL-13 blockade results were highly significant with all 3 techniques, but IL-4 deficiency achieved significance in only 1 assay (Figure 1), and to a lesser degree than IL-13 blockade, emphasizes the potential utility of reducing tissue fibrosis through the inhibition of IL-13.

Moreover, in vitro studies with $3 \mathrm{~T} 3$ cells may demonstrate for the first time the ability of IL-13 to stimulate collagen production in fibroblasts (Figure 5). Thus, the effects of IL-13 on fibrosis are likely direct, and not dependent upon modulations in the Th1/Th2 cytokine response. In support of this conclusion, recent studies identified IL-13 receptors on fibroblasts (22) and exogenous IL-13 increased adhesion molecule and inflammatory cytokine expression in human lung fibroblasts (41). Additional studies are needed however, to determine whether granuloma-derived fibroblasts exhibit similar activity. Finally, although IL-13 (Figure 5) and IL-4 (42) are both capable of promoting collagen production in fibroblasts, the fact that cultured lymph node cells produced nearly 100 -fold more IL-13 than IL-4 (Figure 2) only serves to emphasize the potentially important contribution of IL-13 in this process. Indeed, studies in the pulmonary granuloma model revealed that IL-4 mRNA expression is more tightly regulated at the site of lesion formation, whereas the induction of IL-13 mRNA is much more sustained over time (29) and, similar to the findings reported here, is observed at greater levels than IL-4 (8). Nevertheless, we have not examined the kinetics of IL-4 and IL-13 mRNA expression in infected animals, so we cannot say whether a similar pattern holds in the granulomatous livers.

IL-13 was also recently shown to play a central role in murine asthma $(30,31)$. Indeed, the targeted pulmonary expression of IL-13 was shown to cause mucus cell metaplasia, airway fibrosis, and nonspecific airway hyperreactivity (43). Subepithelial fibrosis and airway smooth muscle hypertrophy are common features of chronic severe asthma (44), and chronic pulmonary fibrosis is associated with the production of collagen I and collagen III. Thus, the link between IL-13 and fibrosis revealed in our study may extend to other important Th2-related human diseases and may lead to more effective modes of treatment of fibrotic disease in general. In summary, our findings provide evidence that IL-13 inhibitors, such as the sIL-13R $\alpha 2-F c$, may be of general therapeutic benefit in preventing fibrosis associated with chronic infectious dis- 
ease, and demonstrate the important and nonredundant role of IL-13 in the pathogenesis of schistosomiasis.

\section{Acknowledgments}

We would like to thank Lori Fitz, Richard Zollner, and Jenifer Thomas for production and purification of recombinant sIL-13R $22-\mathrm{Fc}$; Fred Lewis (Biomedical Research Institute) for providing SEA and cercariae of $S$. mansoni; and Ron Germain for providing the mouse 3T3 fibroblast cell line. We are also grateful to Karl Hoffmann, Matthias Hesse, Alan Sher, and Joe Sypek for their helpful comments and suggestions.

1. Rosenstein, B.J., and Zeitlin, P.L. 1998. Cystic fibrosis. Lancet. 351:277-282

2. Trojanowska, M., LeRoy, E.C., Eckes, B., and Krieg, T. 1998. Pathogenesis of fibrosis: type 1 collagen and the skin. J. Mol. Med. 76:266-274.

3. Johnson, L.L., Dyer, R., and Hupe, D.J. 1998. Matrix metalloproteinases. Curr. Opin. Chem. Biol. 2:466-471.

4. Cheever, A.W., and Yap, G.S. 1997. Immunologic basis of disease and disease regulation in schistosomiasis. Chem. Immunol. 66:159-176.

5. Bergquist, N.R. 1998. Schistosomiasis vaccine development: progress and prospects. Mem. Inst. Oswaldo Cruz. 93:95-101.

6. Grzych, J.M., et al. 1991. Egg deposition is the major stimulus for the production of Th2 cytokines in murine schistosomiasis mansoni. J. Immunol. 146:1322-1327.

7. Pearce, E.J., Caspar, P., Grzych, J.M., Lewis, F.A., and Sher, A. 1992. Downregulation of Th1 cytokine production accompanies induction of Th2 responses by a parasitic helminth, Schistosoma mansoni. J. Exp. Med. 173:159-162.

8. Chensue, S.W., Warmington, K., Ruth, J.H., Lukacs, N., and Kunkel, S.L. 1997. Mycobacterial and schistosomal antigen-elicited granuloma formation in IFN-gamma and IL-4 knockout mice: analysis of local and regional cytokine and chemokine networks. J. Immunol. 159:3565-3573.

9. Cheever, A.W., et al. 1994. Anti-IL-4 treatment of Schistosoma mansoni-infected mice inhibits development of $\mathrm{T}$ cells and non-B, non-T cells expressing Th2 cytokines while decreasing egg-induced hepatic fibrosis. J. Immunol. 153:753-759.

10. Kaplan, M.H., Whitfield, J.R., Boros, D.L., and Grusby, M.J. 1998. Th2 cells are required for the Schistosoma mansoni egg-induced granulomatous response. J. Immunol. 160:1850-1856.

11. Chensue, S.W., et al. 1992. Role of interleukin-4 and gamma-interferon in Schistosoma mansoni egg-induced hypersensitivity granuloma formation: orchestration, relative contribution and relationship to macrophage function. J. Immunol. 148:900-910.

12. Henderson, G.S., Lu, X., McCurley, T.L., and Colley, D.G. 1992. In vivo molecular analysis of lymphokines involved in the murine immune response during Schistosoma mansoni infection. II. Quantitation of IL-4 mRNA, IFN-g mRNA, and IL-2 mRNA levels in the granulomatous livers, mesenteric lymph nodes, and spleens during the course of modulation. $J$ Immunol. 148:2261-2267.

13. Wynn, T.A., et al. 1995. An IL-12-based vaccination method for preventing fibrosis induced by schistosome infection. Nature. 376:594-596.

14. Secor, W.E., Stewart, S.J., and Colley, D.G. 1990. Eosinophils and immune mechanisms. VI. The synergistic combination of granulocyte-macrophage colony-stimulating factor and IL-5 accounts for eosinophil-stimulation promoter activity in Schistosoma mansoni-infected mice. J. Immunol. 144:1484-1489.

15. Le Gros, G., Ben-Sasson, S.Z., Seder, R., Finkelman, F.D., and Paul, W.E. 1990. Generation of interleukin 4(IL-4)-producing cells in vivo and in vitro: IL-2 and IL-4 are required for in vitro generation of IL-4- producing cells. $J$. Exp. Med. 172:921-929.

16. Kopf, M., et al. 1993. Disruption of the murine IL-4 gene blocks Th2 cytokine responses. Nature. 362:245-248.

17. Metwali, A., et al. 1996. The granulomatous response in murine schistosomiasis mansoni does not switch to Th1 in IL-4-deficient C57BL/ 6 mice. J. Immunol. 157:4546-4553.

18. Pearce, E.J., et al. 1996. Schistosoma mansoni in IL-4-deficient mice. Int. Immunol. 8:435-444.

19. Noben-Trauth, N., et al. 1997. An interleukin 4 (IL-4)-independent pathway for CD4+ T cell IL-4 production is revealed in IL-4 receptor-deficient mice. Proc. Natl. Acad. Sci. USA. 94:10838-10843.
20. Barner, M., Mohrs, M., Brombacher, F., and Kopf, M. 1998. Differences between IL-4R alpha-deficient and IL-4-deficient mice reveal a role for IL13 in the regulation of Th2 responses. Curr. Biol. 8:669-672.

21. de Vries, J.E. 1998. The role of IL-13 and its receptor in allergy and inflammatory responses. J. Allergy Clin. Immunol. 102:165-169.

22. Murata, T., Husain, S.R., Mohri, H., and Puri, R.K. 1998. Two different IL13 receptor chains are expressed in normal human skin fibroblasts, and IL4 and IL-13 mediate signal transduction through a common pathway. Int. mmunol. 10:1103-1110.

23. Emson, C.L., Bell, S.E., Jones, A., Wisden, W., and McKenzie, A.N. 1998. Interleukin (IL)-4-independent induction of immunoglobulin (Ig)E, and perturbation of T cell development in transgenic mice expressing IL-13. J. Exp. Med. 188:399-404.

24. McKenzie, G.J., et al. 1998. Impaired development of Th2 cells in IL-13-deficient mice. Immunity. 9:423-432

25. Donaldson, D.D., et al. 1998. The murine IL-13Ra2: molecular cloning, characterization and comparison with murine IL-13Ra1. J. Immunol. 161:2317-2324.

26. McKenzie, G.J., Bancroft, A., Grencis, R.K., and McKenzie, A.N. 1998. A distinct role for interleukin-13 in Th2-cell-mediated immune responses. Curr. Biol. 8:339-342.

27. Bancroft, A.J., McKenzie, A.N., and Grencis, R.K. 1998. A critical role for IL13 in resistance to intestinal nematode infection. J. Immunol. 160:3453-3461.

28. Urban, J.F., Jr., et al. 1998. IL-13, IL-4Ralpha, and Stat6 are required for the expulsion of the gastrointestinal nematode parasite Nippostrongylus brasiliensis. Immunity. 8:255-264.

29. Chiaramonte, M.G., et al. 1999. IL-13 is a key regulatory cytokine for Th2 cell-mediated pulmonary granuloma formation and IgE responses induced by Schistosoma mansoni eggs. J. Immunol. 162:920-930.

30. Wills-Karp, M., et al. 1998. Interleukin-13: central mediator of allergic asthma [see comments]. Science. 282:2258-2261.

31. Grunig, G., et al. 1998. Requirement for IL-13 independently of IL-4 in experimental asthma [see comments]. Science. 282:2261-2263.

32. Hilton, D.J., et al. 1996. Cloning and characterization of a binding subunit of the interleukin 13 receptor that is also a component of the interleukin 4 receptor. Proc. Natl. Acad. Sci. USA. 93:497-501.

33. Miloux, B., et al. 1997. Cloning of the human IL-13R alpha1 chain and reconstitution with the IL4R alpha of a functional IL-4/IL-13 receptor complex. FEBS Lett. 401:163-166.

34. Gauchat, J.F., et al. 1997. A novel 4-kb interleukin-13 receptor alpha mRNA expressed in human B, T, and endothelial cells encoding an alternate typeII interleukin-4/interleukin-13 receptor. Eur. J. Immunol. 27:971-978.

35. Bergman, I., and Loxley, R. 1963. Two improved and simplified methods for the spectrophotometric determination of hydroxyproline. Anal. Biochem. 35:1961-1965.

36. Wynn, T.A., et al. 1993. Analysis of cytokine mRNA expression during primary granuloma formation induced by eggs of Schistosoma mansoni. J. Immunol. 151:1430-1440.

37. Wynn, T.A., Eltoum, I., Oswald, I.P., Cheever, A.W., and Sher, A. 1994. Endogenous interleukin 12 (IL-12) regulates granuloma formation induced by eggs of Schistosoma mansoni and exogenous IL-12 both inhibits and prophylactically immunizes against egg pathology. J. Exp. Med. 179:1551-1561.

38. Warren, K.S. 1982. Schistosomiasis: host-pathogen biology. Rev. Infect. Dis. 4:771-775.

39. Kaplan, M.H., Schindler, U., Smiley, S.T., and Grusby, M.J. 1996. Stat6 is required for mediating responses to IL-4 and for development of Th2 cells. Immunity. 4:313-319.

40. Dunne, D.W., Jones, F.M., and Doenhoff, M.J. 1991. The purification, characterization, serological activity and hepatotoxic properties of two cationic glycoproteins (alpha 1 and omega 1) from Schistosoma mansoni eggs. Parasitology. 103:225-236.

41. Doucet, C., et al. 1998. IL-4 and IL-13 specifically increase adhesion molecule and inflammatory cytokine expression in human lung fibroblasts. Int. Immunol. 10:1421-1433.

42. Serpier, H., et al. 1997. Antagonistic effects of interferon-gamma and interleukin-4 on fibroblast cultures. J. Invest. Dermatol. 109:158-162.

43. Zhu, Z., et al. 1999. Pulmonary expression of interleukin-13 causes inflammation, mucus hypersecretion, subepithelial fibrosis, physiologic abnormalities, and eotaxin production. J. Clin. Invest. 103:779-788.

44. Bento, A.M., and Hershenson, M.B. 1998. Airway remodeling: potential contributions of subepithelial fibrosis and airway smooth muscle hypertrophy/hyperplasia to airway narrowing in asthma. Allergy Asthma Proc. 19:353-358 